\title{
A Brief Introduction in the Mitigation of Conducted Electromagnetic Interference Issues
}

\author{
M. Buzdugan \\ Department of Buildings Engineering \\ Technical University of Cluj-Napoca \\ 28 Memorandumului str., 400114, Cluj-Napoca (Romania) \\ Phone/Fax number: +40 744 560833, e-mail: mircea.buzdugan@insta.utcluj.ro
}

\begin{abstract}
This paper deals with the mitigation of the influence of electromagnetic conducted emissions in low voltage grids, which can be performed using different filtering methods. Due to the relatively young age of the electromagnetic compatibility domain, the specific terminology is not yet fully consecrated. That is why the specific literature abounds in a bunch of definitions and notions, incomplete, redundant, or worse, even contradictory. Therefore, all over this paper, the terminology from the successive issues of the standard IEC 60050-161 International Electrotechnical Vocabulary, is used. The introductory section presents generalities regarding the broader context of electromagnetic compatibility in which the paper fit. Section II is devoted to measurement techniques and measuring equipment used in conducted electromagnetic interference tests, specifically for electromagnetic emissions that flow in/from the equipment under test through power lines in the standardized frequency range from $100 \mathrm{kHz}$ to $30 \mathrm{MHz}$. These measurement techniques and equipment are further used in the next section which presents electromagnetic interference experiments, performed on an induction motor driven by a frequency converter. To mitigate the conducted electromagnetic emissions to fit into the standard limits, a cascade of two EMI filtering cells has been designed and implemented. This demonstrated the usefulness and effectiveness of mains EMI filters in low voltage power applications. The experiment also demonstrated that in some cases it would be necessary to retrofit more than one filtering cell.
\end{abstract}

Key words. electromagnetic compatibility, conducted electromagnetic interference, line impedance stabilization network, spectrum analyzer, EMI passive mains filter.

\section{Introduction}

According to its definition, electromagnetic compatibility, EMC, means the ability of an equipment or system to function satisfactorily in its electromagnetic environment without introducing intolerable electromagnetic disturbances to anything in that electromagnetic environment [1].

The electromagnetic environment means the totality of electromagnetic phenomena existing at a given location. In general, the electromagnetic environment is time dependent, and its description may need a statistical approach.
As it can be seen from the above definitions, the terms "satisfactory" and "intolerable" as well as the syntagma "electromagnetic environment" express a kind of ambiguity and indicate the dependence on a certain situation, indicating somehow the complexity of the domain, which very often cannot be shipped through a few standard limit values. Beyond the qualitative aspect of the definition, one must admit that the concept of electromagnetic compatibility, in all its aspects, insinuates more and more seriously in the general concept of quality of life.

Lack of electromagnetic compatibility, or a poor solution to its problems may have many consequences, from minor irritations caused by an interrupted or bad quality of audio/video reception to unpredictable or unreliable operation of equipment in residential, commercial, or industrial environment culminating to risks upon the ecosystem and living beings. In this respect see the concept of biocompatibility, for details [2].

Standard IEC 60050-161 International Electrotechnical Vocabulary, Chapter 161: Electromagnetic compatibility, defines also the approximately similar notions of electromagnetic disturbance (an electromagnetic noise, an unwanted signal or a change in the propagation medium itself), as any electromagnetic phenomenon which may degrade the performance of a device, equipment or system, or adversely affect living or inert matter and of electromagnetic interference, as the degradation of the performance of an equipment, transmission channel or system caused by an electromagnetic disturbance. The terms "electromagnetic disturbance" and "electromagnetic interference" designate the cause and respectively the effect, but they are often used indiscriminately.

The specific literature classifies the domain of electromagnetic compatibility on different criteria. A first criterion which I would call "technological" distinguishes between the terms of inter-system interference, i.e. the electromagnetic interference in one system due to an electromagnetic disturbance produced by another system and intra-system interference, i.e. the electromagnetic interference a phenomenon occurring in a system due to 
an electromagnetic disturbance produced within the same system, often designated as self-interference.

In other criteria of classification, electromagnetic interference is designated as reversible/irreversible, respectively natural (having its source in natural phenomena and not generated by man-made devices) and man-made (having its source in man-made devices), the latest being in its turn intentional/unintentional

Finally, in EMC regulations, the mechanisms of electromagnetic interference are divided into two large categories, conducted, and radiated.

In conducted interference, the high frequency energy may couple to the power supply and "escape" out of an electrical equipment, through the power supply cord or other signal cables.

On the other hand, radiated interference (term sometimes used to cover induction phenomena as well) represents the electromagnetic disturbance for which the energy is transferred through space in the form of electromagnetic waves. High frequency signals that "escape" from an equipment via the mains or signal port may also radiate quite readily.

Speaking of radiated interference, nor the standards and unfortunately in many cases neither the specific literature distinguishes between near field radiation and far field radiation, both being embedded in the same category of radiated emissions. While those who deal with electromagnetism are familiar with the difference between near-field coupling and far-field emissions, the vague terminology in EMC regulations tends readily to lead to confusions.

An alternative term often used for interference is the electromagnetic emission, the phenomenon by which electromagnetic energy exits from a source by way of conduction or radiation mechanisms.

Conducted emissions are controlled mainly by filtering techniques and proper grounding practices, while radiated emissions are controlled by shielding procedures.

In contrast to emissions, electromagnetic immunity represents the ability of a device, equipment, or system to perform without degradation in the presence of an electromagnetic disturbance. Immunity tests the capacity of equipment and systems to withstand the two categories of emissions mentioned above.

Electromagnetic susceptibility is a complementary notion to immunity, being simple the lack of immunity and representing the inability of a device, equipment or system to perform without degradation in the presence of an electromagnetic disturbance.

Fig. 1 depicts a simplified tree structure classification of electromagnetic compatibility. One can observe that, in the electromagnetic immunity branch of the tree, is inserted a new notion, namely the electrostatic discharges
(ESD), the transfer of electric charges between bodies of different electrostatic potential in proximity or through direct contact.

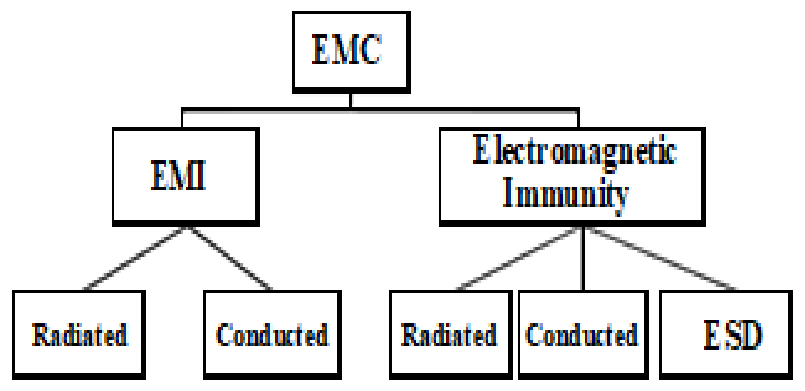

Fig. 1 Simplified tree structure of electromagnetic compatibility mechanisms

\section{Equipment and Techniques for Measuring Conducted Interference}

Presumably, one of the most complex aspects of electromagnetic compatibility is the multitude of techniques involved in performing specific measurements.

In terms of frequency, EMC phenomena extend from low frequencies, from low frequency, say above the 100 order harmonics approximately above $5 \mathrm{kHz}$, until above high frequencies of $3 \mathrm{GHz}$, which makes the use of conventional measurement techniques quite irrelevant.

For ease of measurement and analysis, radiated emissions are assumed to predominate above $30 \mathrm{MHz}$ while conducted emissions are assumed to predominate below $30 \mathrm{MHz}$. Of course, anything magic can happen at 30 $\mathrm{MHz}$, but cables with typical lengths for these frequencies tend to excite resonant frequencies above $30 \mathrm{MHz}$, leading to abnormal measurement results. At the same time, measurements of radiated fields at frequencies below 30 $\mathrm{MHz}$, performed in near field, always concentrated near the source, at less than $\lambda / 2 \pi$, being a storage reactive field and not a propagating one, lead to results which will be not correlated to reality.

The primary instrument for EMC measurements is an EMC receiver that covers the frequency range from 100 $\mathrm{kHz}$ to $3 \mathrm{GHz}$ and even beyond. The distinguishing features of such a measuring receiver in relation to a standard spectrum analyzer are [3]:

- much better sensitivity, allowing signals to be discriminated from noise at levels well below the emission limits;

- better robustness of input circuits and higher withstanding level to overload;

- special design for measuring to CISPR standards, with bandwidths, detectors and dynamic range of the signal circuit adapted for this purpose;

- better accuracy of the frequency and amplitude than that of ordinary spectrum analyzers;

In measurements performed in the next section, a spectrum analyzer is used, namely a measuring equipment 
widely used for "quick-look" testing and diagnostics, provided supplementary with a tracking generator.

The spectrum analyzer model used in the following experimental measurements in this paper is HM5014 having embedded a tracking generator, that can be used to evaluate frequency characteristics of quadrupoles, such as passive EMI filters. The spectrum analyzer is controlled by the SW 5012E-V147 firmware, via an RS-232 serial interface.

The electromagnetic emissions generated by an equipment power connected and conducted in the supply lines of the mains, must be analyzed using a line impedance stabilization network (LISN), and the spectrum analyzer/EMC receiver, the equipment under test (EUT) being connected directly to the LISN [5]. See also Fig. 4.

In principle, the impedance stabilization network is a low pass filtering network, which connect the EUT to the a.c. power lines, providing a quite pure sinusoidal waveform of the supply voltage.

A first goal of LISN is to provide the power cord of the EUT with a constant impedance socket throughout the frequency range of the conducted emission tests.

For measurements accuracy, the second goal of the LISN is to block conducted emissions that are not due to the equipment under test. Obviously, LISNs are built for different levels of power.

Measurement techniques distinguish between three types of impedance stabilization networks [1]:

artificial mains network (line impedance stabilization network), LISN (abbreviation) (USA): a network inserted in the supply mains cable of equipment under test which provides, in a certain frequency range, a specified load impedance for the measurement of disturbance voltages and which may isolate the apparatus from the supply mains in that frequency range.

- delta network: an artificial mains network enabling to test separately the common mode and differential mode voltages of a single-phase circuit.

- V-network: an artificial mains network enabling the voltages between each conductor and earth to be measured separately. The V-network may be designed for applications to grids having any number of conductors.

Fig. 2 depicts a V- network artificial mains network, used in the next experimental section of the present paper.

Considering the output of a LISN, as standardized impedances of $50 \mathrm{~Hz}$, the analysis of conducted interferences is greatly simplified, as in Fig. 3.

In signals transmission that uses supply cables as a transmission channel, the problem of electromagnetic interference has always been a cause for concern.

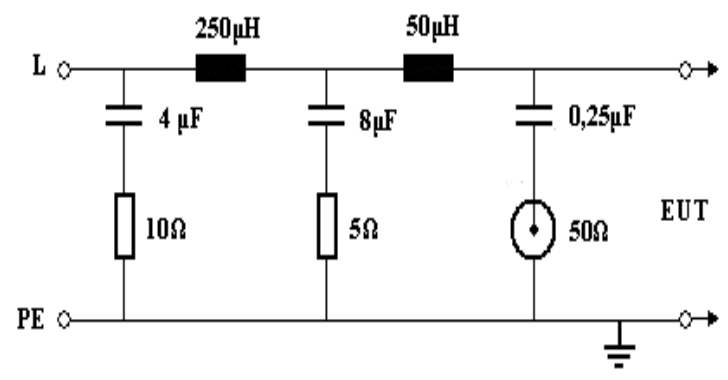

Fig. 2 V-network type artificial mains network

Apart from the electromagnetic noise generated by printed circuit boards, power and signal cables are considered also main transmission mediums.

On the other hand, transformers, common mode shocks and autotransformers can play a key role in mitigating electromagnetic disturbance emissions in power and signal transmission networks, as well as in their interfaces, due to their predominant inductance.

In the analysis of conducted electromagnetic emissions, differential and common mode currents represent an essential dichotomy.

In a two-conductor cable, or simply for any two particular conductors in a multi-conductor cable, half the magnitude of the difference of the phasors representing the currents in each conductor is the differential mode current, while in a cable having more than one conductor, including shields and screens if any, the magnitude of the sum of the phasors representing the currents in each conductor is the common mode current.

Be it an equipment (Fig. 3), supplied by low voltage, the supply grid situated in a radiofrequency environment. The equipment is shielded by a metal housing being provided with a conductor for PE protective neutral. It is important to mention that in the radiofrequency range even in the absence of the PE conductor, there will be a return current path through the parasitic capacity with respect to the ground.

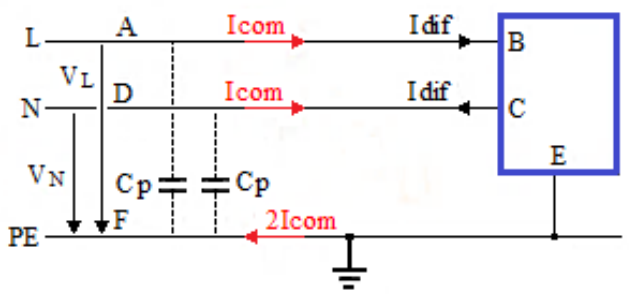

Fig. 3 LISN equivalent circuit seen by the equipment

In Fig. 3 three current flow loops can be defined as:

- Loop 1: ABCDA consisting of the phase $\mathrm{L}$ and the neutral $\mathrm{N}$ supply conductors and the equipment through which the Idif current flows in differential mode.

- Loop 2: ABEFA consisting of the phase conductor $\mathrm{L}$ and the protective neutral conductor PE through which the Icom current flows in common mode. 
- Loop 3: DCEFD consisting of the neutral conductor $\mathrm{N}$ and the protective neutral conductor PE through which the Icom current flows also in common mode.

As Fig. 3 suggests, the common mode currents seek to close using parasitic capacitances, the common mode disturbances always having higher values and more insidious effects than the differential mode ones, since the common mode loops are always larger than in differential mode. The differential mode disturbances occur only due to asymmetries and non-uniformity of circuits.

The radiofrequency electromagnetic field RF is coupled in common mode in the two supply lines, and in the ideal case of a perfect symmetrical geometry of the circuits would not cause differential disturbances.

The effect of disturbances caused by RF fields in low voltage networks depends on the RF properties at both extremities of the supply conductors, on the values of capacitances and inductances distributed along them, and on the nature of the internal and external grounding paths of the equipment.

Loop 1 (ABCDA) shows that differential disturbances will produce a voltage at the circuit terminals, which can often be benign if it does not exceed certain limits.

Examination of loops 2 (ABEFA) and 3 (DCEFD) shows that common mode disturbances will seek for a closing path to the ground. However, if there is no local low impedance closing path, common mode disturbing currents will flow to ground through parasitic capacitances distributed along signal and ground paths in printed circuits, causing harmful signal modulation and faulty operation, culminating even with the destruction of circuits, especially in the case of sensitive electronic ones.

Using a quantitative approach, according to Fig. 3 [4]:

$I_{L}=I_{c o m}+I_{d i f}$

$I_{L}=I_{\text {com }}-I_{d i f}$

It results that:

$I_{\text {dif }}=\frac{1}{2}\left(I_{L}-I_{N}\right)$

$I_{\text {com }}=\frac{1}{2}\left(I_{L}+I_{N}\right)$

Considering the outputs of the LISN as $50 \Omega$ impedances, the input voltages in the EUT will be:

$V_{L}=50\left(I_{\text {com }}+I_{d i f}\right)$

$V_{N}=50\left(I_{c o m}-I_{\text {dif }}\right)$

It is easy to observe that in the case of electromagnetic conducted emissions the common mode currents can be of the same order as differential mode currents, but most of the time they are much higher than the differential mode currents. This observation is the basis of the principle of operation of the EMI filters elements of circuit, namely those filters that help to mitigate or, if possible, eliminate conducted electromagnetic emissions (i.e. common mode choke, common mode and differential mode capacitors) [6].

Fig. 4 shows the block diagram of a test setup for electromagnetic interference generated by the EUT in the supply lines in the frequency range of interest of $100 \mathrm{kHz}$ - $30 \mathrm{MHz}$.

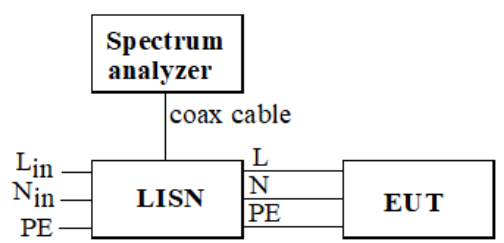

Fig. 4 Block diagram of a conducted EMI test setup

The spectrum analyzer, controlled by its firmware, downloads in an associated computer the graphs of the instantaneous, quasi-peak and average values of conducted electromagnetic emissions, using obviously the appropriate embedded detectors.

A quasi-peak detector is a device having specified electrical time constants which, when regularly repeated identical pulses are applied to it, delivers an output voltage which is a fraction of the peak value of the pulses, the fraction increasing towards unity as the pulse repetition rate is increased. On the other hand, an average detector detects the output voltage of which is the average value of the envelope of an applied signal. The average value must be taken over a specified time interval.

The graphs are represented in a logarithmic measurement unit, respectively $\mathrm{dB} \mu \mathrm{V}$ :

$\mathrm{dB} \mu \mathrm{V} \triangleq 20 \log _{10}\left(\frac{\text { volts }}{1 \mu \mathrm{V}}\right)$

EMI filters are characterized by their insertion loss, IL, defined by the ratio of the power consumed by the load in a reference circuit before and after the filter retrofit into a circuit.

The firmware that controls the tracking generator, displays the attenuation characteristic of the input signal, which is the symmetrical (inverse) characteristics of the insertion loss characteristics, as it can be seen in Fig. 7.

Figs. 5 and 6 show the block diagrams of the setups used in obtaining the specific characteristics of the EMI filters in differential and common mode. The experimental setup is presented in Fig. 7.

For all measurements performed, a reference-ground plane (US) must be used, namely a flat conductive surface whose potential is used as a common reference. One can observe in Fig. 7 the compulsory reference ground plane in silver fabric in this situation.

The practical setup for obtaining the graphs of these features is shown in Fig. 7. 


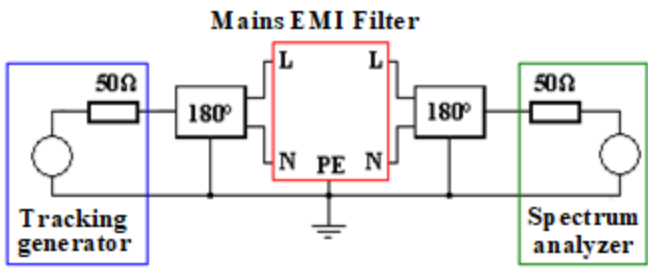

Fig. 5 Measurement setup to obtain the attenuation characteristics of EMI filters in differential mode

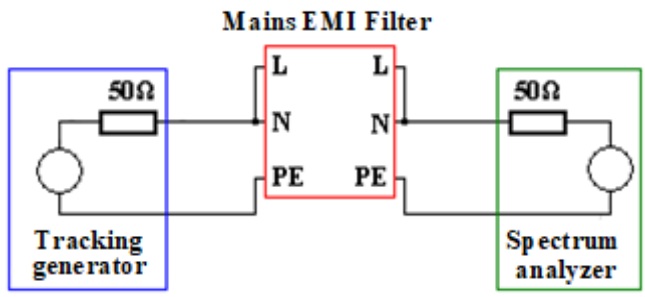

Fig. 6 Measurement setup to obtain the attenuation characteristics of EMI filters in common mode

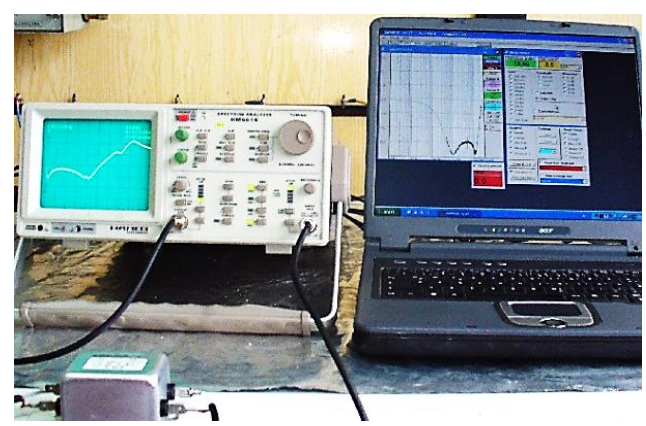

Fig. 7 The setup used for displaying EMI filters attenuation characteristics.

\section{Experimental Results}

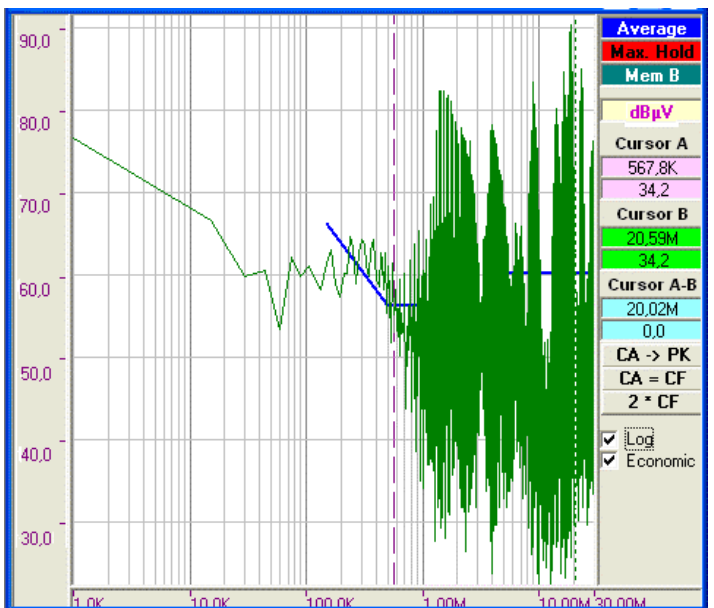

Fig. 8 Electromagnetic emissions conducted through the power cord of the equipment

Next, in this section, a short overview of passive EMI mains filters will be addressed, presenting the necessity and effectiveness of their use.

The conducted electromagnetic emissions mitigation experiment was performed on an equipment consisting of a three-phase squirrel cage induction motor, wye connected, having the rated power $P_{n}=360 \mathrm{~W}$. The motor is driven by a frequency converter of $1.5 \mathrm{~kW}$, powered from the 230 Vac single-phase low voltage public network.

Fig. 8 depicts the conducted electromagnetic interference that propagates through the $230 \mathrm{~V}$ low voltage supply lines from the EUT. The measurements were performed using the setup whose block diagram is depicted in Fig. 4.

Since mains EMI filters carry potentially high currents and potentially dangerous voltages, great care must be taken in their design.

First, the operating voltage and the current flowing through the components must be known, the basic specification including also mechanical details such as the housing size, fastening methods and weight limits.

At the same time, the electrical specification, must comply with national and international electrical safety standards.

In addition, EMC performance, usually the frequencydependent insertion attenuation curve and the leakage current must be specified as well. For medical equipment, especially those patients connected, it is compulsory to know the limits of the leakage current [7].

EMI passive filters must ensure a high degree of mismatch in the characteristic impedance "seen" by a disturbing (unwanted) signal, blocking them both in differential and common mode, the main point being to reflect most of this disturbing energy back to the source that generated it, and less to dissipate it inside the filter, thus determining its uncontrolled overheating.

It should be noted that EMI filters are electrically reciprocal structures. This feature allows them to be two way mitigating device, capable to block the two-way electromagnetic conducted emissions, respectively those generated by the equipment and those incoming from the supply lines.

From the characteristics presented in Fig. 8, one can see that the standard limits are exceeded especially in the frequency range from $1 \mathrm{MHz}$ to $10 \mathrm{MHz}$. Consequently, several filtration variants were designed, but after the tests the need to introduce two EMI filter cells cascade connected, as Fig. 9 reveals became obvious.

One may see in both filtering cells the compulsory typical components, namely the common mode choke and the common and differential mode mitigation capacitors $\mathrm{Cy}$ and $\mathrm{Cx}[8]$.

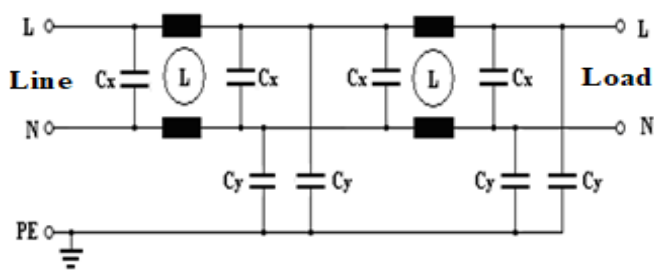

Fig. 9 Cascade of two passive EMI filters 
The insertion attenuation characteristics of the two filtering cells belonging to the cascade are depicted on the same graph in Fig. 10. One can see the importance of the secondary filtering cell (blue line) in the upper range around $10 \mathrm{MHz}$. The retrofitting of a second supplementary cell was compulsory.

Fig. 11 depicts the attenuation characteristics of the filtering cascade. Both characteristics are logarithmically displayed, better highlighting the interest area between $1 \mathrm{MHz}$ and $10 \mathrm{MHz}$.

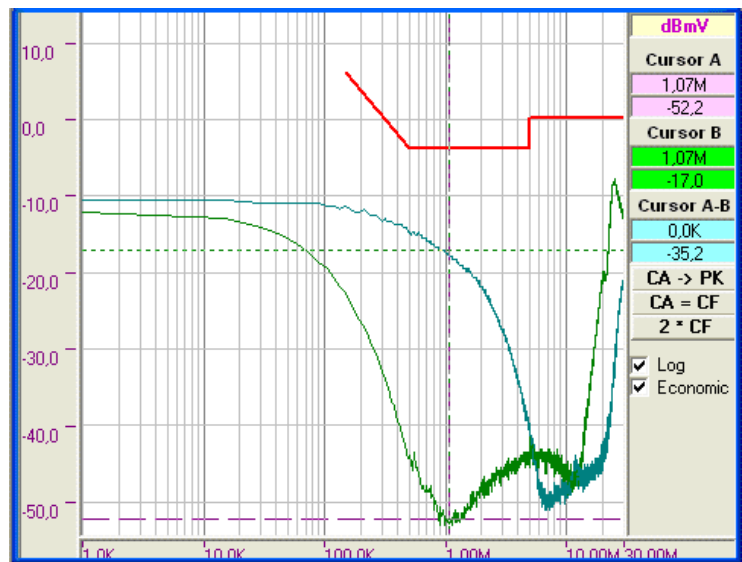

Fig. 10 Insertion attenuation characteristics of the two filters

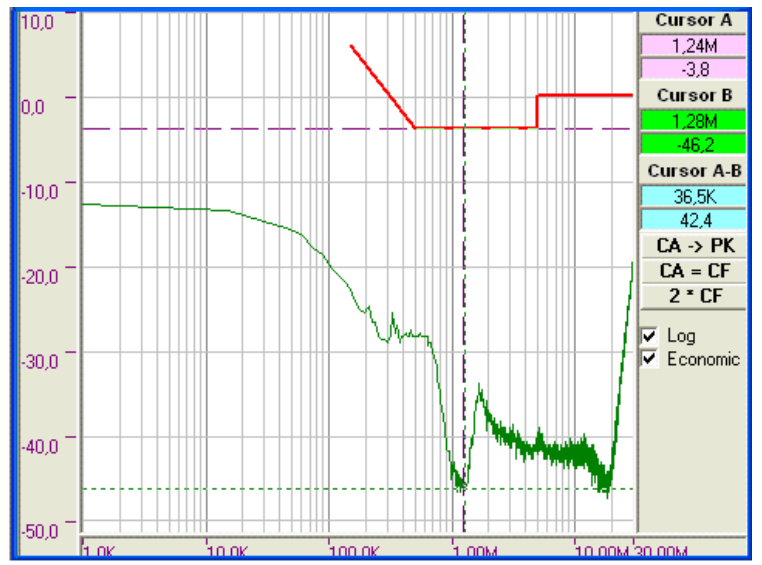

Fig. 11 The two-filters cascade insertion attenuation characteristics

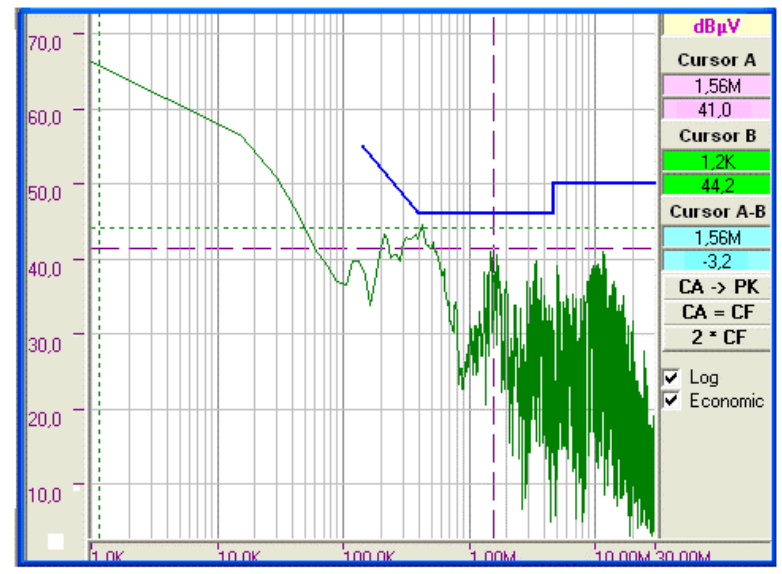

Fig. 12. Electromagnetic emissions led through the power cord after retrofitting the EMI filters cascade connected.

One can see that if prior the introduction of the cascade the standard limits were exceeded by $30 \mathrm{~dB} \mu \mathrm{V}$, after the retrofit of the cascade, the values of electromagnetic conducted emissions have decreased from approximately $90 \mathrm{~dB} \mu \mathrm{V}$ to $44.2 \mathrm{~dB} \mu \mathrm{V}$, namely with $45.8 \mathrm{~dB} \mu \mathrm{V}$, which demonstrates the effectiveness of the solution adopted. Moreover, in the frequency range around $10 \mathrm{MHz}$, the decrease is more pronounced, the maximum recorded values being only of $40 \mathrm{~dB} \mu \mathrm{V}$.

It must be noted that the electromagnetic emission limits for both IEC standards concerning residential, commercial and light-industrial and industrial environments are fulfilled as it can be seen also in Fig. 12. [9], [10]

\section{Conclusions}

The paper aims to be a brief introduction in the mitigation techniques of electromagnetic conducted emissions into and from single-phase equipment, having rated currents up to $16 \mathrm{~A}$. Passive EMI filtering is one of the most common methods in this matter.

The need for passive filtration arises because the producers of different equipment, design and manufacture them usually in standard laboratory conditions, which generally differ from the real ones.

However, very often, due to the real electromagnetic conditions, especially to various electromagnetic induction phenomena that may occur locally, the standard limits may be easily exceeded.

Therefore, retrofitting one or even more filtering cells becomes compulsory, as it could be observed in the paper.

\section{References}

[1] IEC 60050-161 International Electrotechnical Vocabulary, Chapter 161: Electromagnetic compatibility

[2] M.I. Buzdugan, H. Bălan, D. Mureşan, Electromagnetic Compatibility versus Electromagnetic Biocompatibility, 14th International Power Electronics and Motion Control Conference, EPE-PEMC 2010, Ohrid, North Macedonia

[3] T. Williams, EMC for Product Designers, $5^{\text {th }}$ Edition, Elsevier, 2016

[4] C.R. Paul, Introduction to Electromagnetic Compatibility, Second Edition, John Wiley \& Sons, Inc., 2006.

[5] H. W. Ott, Electromagnetic Compatibility Engineering, John Wiley \& Sons, Inc., 2009

[6] K. Kostov, J. Kyyrä, Common-mode choke coils characterization, in Proceedings of the 13th European Conference on Power Electronics and Applications (EPE 2009). Barcelona, Spain. 8-10 September 2009.

[7] IEC 60601-2, Edition 4.0 2014-02, Medical electrical equipment - Part 1-2: General requirements for basic safety and essential performance - Collateral Standard: Electromagnetic disturbances - Requirements and tests

[8] R.L. Ozenbaugh, T.M. Pullen, EMI Filter Design, 3rd Edition, CRC Press, 2017

[9] IEC 61000-6-3, Edition 2.1 2011-02, Electromagnetic compatibility (EMC) -Part 6-3: Generic standards Emission standard for residential, commercial and lightindustrial environments

[10] IEC 61000-6-4, Edition 2.1 2011-02, Electromagnetic compatibility (EMC) -Part 6-3: Generic standards Emission standard for Industrial Environments 